\title{
Clinical Presentation of Left Ventricular Noncompaction Cardi- omyopathy and Bradycardia in Three Families Carrying HCN4 Pathogenic Variants
}

\author{
Agata Paszkowska 1,t, Dorota Piekutowska-Abramczuk ${ }^{2,+}$, Elżbieta Ciara ${ }^{2}$, Alicja Mirecka-Rola ${ }^{1}$, Monika \\ Brzezinska ${ }^{1}$, Dorota Wicher ${ }^{2}$, Grażyna Kostrzewa ${ }^{3}$, Jędrzej Sarnecki ${ }^{4}$ and Lidia Ziółkowska 1,* \\ 1 Department of Cardiology, The Children's Memorial Health Institute, 04-730 Warsaw, Poland \\ 2 Department of Medical Genetics, The Children's Memorial Health Institute, 04-730 Warsaw, Poland \\ 3 Department of Medical Genetics, Medical University of Warsaw, 02-106 Warsaw, Poland \\ 4 Department of Diagnostic Imaging, The Children's Memorial Health Institute, 04-730 Warsaw, Poland \\ * Correspondence: 1.ziolkowska@ipczd.pl; Tel. + 4822 8157370, Fax. +48 228157365 \\ + These authors contributed equally to this work.
}

\begin{abstract}
Background: Left ventricular noncompaction (LVNC) is a genetically and phenotypically heterogeneous cardiomyopathy characterized by a two-layered myocardium consisting of compacted and noncompacted segments, prominent ventricular trabeculations, and intertrabecular recesses. Patients with LVNC are at increased risk to develop heart failure, atrial and ventricular arrhythmias, and/or systemic thromboembolic events. Familial forms of primary sinus bradycardia have been attributed to mutations in HCN4. There are very few reports about the association between HCN4 mutations and LVNC. The aim of our study was to characterize the clinical phenotype of families with LVNC and sinus bradycardia caused by mutation of the HCN4 gene. Methods: From March 2008 to July 2021 we prospectively enrolled 6 patients from 4 families with diagnosed isolated LVNC based on the clinical presentation, family history and echocardiographic and cardiovascular magnetic resonance (CMR) evidence of LVNC. Next generation sequencing (NGS) analysis was undertaken for evaluation of the molecular basis of the disease in each family. Results: A total of 6 children (median age 11 years) were enrolled and followed prospectively for the median of 12 years. All 6 patients were diagnosed with LVNC by echocardiography and 5 participants additionally by CMR. The presence of LGE was found in 3 children. Sinus bradycardia and dilation of the ascending aorta occurred in 5 studied patients. In 4 patients from 3 families the molecular studies demonstrated the presence of rare heterozygous HCN4 mutations. Conclusion: (1) The HCN4 mutation influences the presence of a complex LVNC phenotype, sinus bradycardia and dilation of the ascending aorta. (2) HCN4 mutation may be associated with the early presentation of clinical symptoms and the severe course of the disease. (3) It is particularly important to assess myocardial fibrosis not only within the ventricles, but also in the atria in patients with LVNC and sinus bradycardia.
\end{abstract}

Keywords: left ventricular noncompaction; cardiomyopathy; sinus bradycardia; HCN4 mutation; late gadolinium enhancement; children

\section{Introduction}

Left ventricular noncompaction (LVNC) is a genetically and phenotypically heterogeneous cardiomyopathy characterized by a two-layered myocardium consisting of compacted and noncompacted segments, prominent ventricular trabeculations, and intertrabecular recesses. Frequency of LVNC has increased and it has been reported as comprising $9 \%$ of all childhood

cardiomyopathies in recent studies [1,2]. This form of cardiomyopathy may be sporadic or familial. Patients with LVNC are at increased risk to develop heart failure, atrial and ventricular arrhythmias, and/or systemic embolic events, both in pediatric and adult 
populations $[3,4]$. The spectrum of arrhythmias is very wide, with the most common one being ventricular or supraventricular arrhythmias and the Wolff-Parkinson-White (WPW) syndrome. Although less common, patients with LVNC have been reported to demonstrate a wide variety of bradyarrhythmias including sinus bradycardia, sick sinus syndrome and varying degrees of atrioventricular (AV) block including complete heart block $[5,6,7]$.

In the literature there have been reports of children with LVNC in association with sinus bradycardia, suggesting the possibility that the two diseases may have a common cause [8,9]. Although familial forms of primary sinus bradycardia are recognized, they have been attributed to mutations in HCN4, SCN5A and ANK2. However, there are very few reports in the literature on the association between HCN4 mutations and LVNC [10] .

The aim of our study was to characterize the clinical phenotype of families with LVNC and sinus bradycardia caused by mutation of the HCN4 gene.

\section{Materials and Methods}

\subsection{Study patients}

From March 2008 to July 2021 we prospectively enrolled 6 patients from 4 families with diagnosed LVNC hospitalized in the Department of Cardiology of the Children's Memorial Health Institute. The criteria for inclusion in the study were age $<18$ years at the time of LVNC diagnosis on the basis of clinical presentation (history, symptoms, sinus bradycardia in electrocardiogram, and familial occurrence of LVNC) and echocardiographic evidence of isolated LVNC defined as: (1) The presence of a two-layer structure with a compacted (C) and noncompacted (NC) myocardial layer of trabecular meshwork with deep endomyocardial spaces. (2) A maximal end-systolic ratio of NC/C layers of $>2$. (3) Color Doppler evidence of deep perfused intertrabecular recesses [11]. Only patients with sinus bradycardia diagnosed according to the criteria recommended in the literature were included in the study $[12,13]$. The exclusion criteria from the study were the presence of congenital heart disease, other forms of cardiomyopathy, or neuromuscular disorders.

The Institutional Ethics Committee approved this study. Informed consent was obtained from all individual participants included in the study.

\subsection{Data collection}

Patients' demographics, clinical symptoms, family history of cardiomyopathies and sudden cardiac death (SCD) as well as the results of echocardiography, 12-lead resting ECG with assessment of heart rate (HR), 24-hour Holter ECG with analysis of minimal, maximal, average HR, occurrence of sinus pauses (RR pauses) $>2$ seconds and cardiovascular magnetic resonance (CMR) were collected. In all children, NYHA functional class and clinical symptoms such as chest pain, arrhythmias, syncope, and thromboembolic events were evaluated. Serum NT-proBNP level was assessed in all patients. Each patient underwent genetic blood tests for evaluation of the molecular basis of the disease.

\subsection{Echocardiographic studies}

Echocardiographic imaging was performed using a Philips Epiq7 (Philips Medical Systems, Bothell, WA). Two-dimensional, Doppler, and M-mode echocardiography were performed at rest using standard methods. Echocardiographic images, including parasternal long- and short-axis and apical two-, three-, and four-chamber views were obtained and reviewed by cardiologists certified in echocardiography. Echocardiographic measurements were reviewed based on Jenni's criteria [11]: a ratio of LV NC to C myocardial layer of 2.0 or greater, measured in the parasternal short-axis view in end-systolic phase below the papillary muscle. Echocardiographic measurements included LV end-diastolic diameter (LVEDd), left ventricular ejection fraction (LVEF) according to the Simpson's method. These parameters were evaluated for each patient and indexed to the patient's BSA according to Du Bois' formula. Moreover, z-scores were calculated using the formula 
for z-scores reported in the literature $[14,15]$. LV enlargement was defined as a $z$-score greater than 2 and impairment of systolic function when LVEF $<55 \%$. Measurements of the ascending aorta were taken in the parasternal long-axis view and indexed to the patient's BSA. Ascending aorta enlargement was defined as z-score > 2 [16].

\subsection{Cardiovascular magnetic resonance imaging}

CMR imaging was performed using a 1.5-T magnetic resonance scanner (Magnetom AvantoFit, Siemens, Erlangen, Germany), with a dedicated cardiac phased array coil and electrocardiographic (ECG) gating. Steady-state free precession (SSFP) cine images of the heart were acquired in the standard views, i.e. in the short-axis and 2-, 3- and 4- chamber planes with a minimum of 25 phases/cardiac cycle. Late gadolinium enhancement (LGE) images were acquired in the short- and long-axis planes, 10-15 minutes after intravenous administration of $0.1 \mathrm{mmol} / \mathrm{kg}$ of gadobutrol (Gadovist, Bayer, Berlin, Germany). The studies were analyzed on a dedicated diagnostic workstation using CVi42 (Circle, Calgary, Canada) software. LVNC was diagnosed based on Petersen's criteria [17], if N/C myocardial thickness ratio, measured in diastole, was $>2.3$. Left ventricular volumes and ejection fraction were assessed semi-automatically based on the short-axis images using the Simpson's method, with papillary muscles excluded from the volumes and included in the myocardial mass [18]. The results were interpreted with z-scores' determination using the multicenter pediatric reference values for CMR-derived LV parameters [19]. Left ventricular LGE was visually evaluated and diagnosed if there were areas of hyperintensity in the myocardium visible in two distinct projections.

\subsection{Molecular studies}

DNA from the peripheral blood was extracted automatically by MagCore Nucleic Acid Extractor HF16Plus (RBC Bioscience) according to the manufacturer's protocol. Candidate gene sequencing was performed on the HiSeq 1500 platform (Illumina) using the TruSight One Sequencing Panel (Illumina) in Family 1, and the original CMHI pediatric panel of 1000 clinically relevant genes (Roche) in the remaining families. A set of 222 and 165 cardiomyopathy-associated genes were analyzed, respectively (Supplementary Material). A detailed study protocol has been described previously [20].

The discovered variants were analyzed and prioritized considering: $1 /$ the minor allele frequency (MAF) determined with the Genome Aggregation Database (gnomAD, https://gnomad.broadinstitute.org/), Exome Variant Server (EVS, https:// evs.gs.washington.edu/EVS/), UK10K Project (https://www.uk10k.org/), and in-house database comprising > 5000 individuals (Department of Medical Genetics; DMG); 2/ the pathogenicity of the variants, using in silico prediction algorithms: CADD, SIFT, MutationTaster, PolyPhen2_HDIV, PolyPhen2_HVAR, MutationAssessor, MetaSVM, MetaLR, and FATHMM; 3/ phenotypic descriptions in Online Mendelian Inheritance in Man (OMIM, https://www.ncbi.nlm.nih.gov/omim), the Human Gene Mutation Database (HGMD, http://www.hgmd.cf.ac.uk), ClinVar (https://www.ncbi.nlm.nih.gov/clinvar/), and Pubmed.

All variants were confirmed by Sanger sequencing and reported with the HCN4 NM_005477.3 (NP_005468.1) reference sequences. Sanger sequencing was also used for parental segregation analysis.

\section{Results}

Baseline characteristics. A total of 6 children from 4 families were enrolled and followed prospectively for the median of $12(6-13)$ years. The median age of patients was 11 (6 - 16) years. Clinical characteristics of the study patients are presented in Table1. 
Table 1. Baseline characteristics of the study patients.

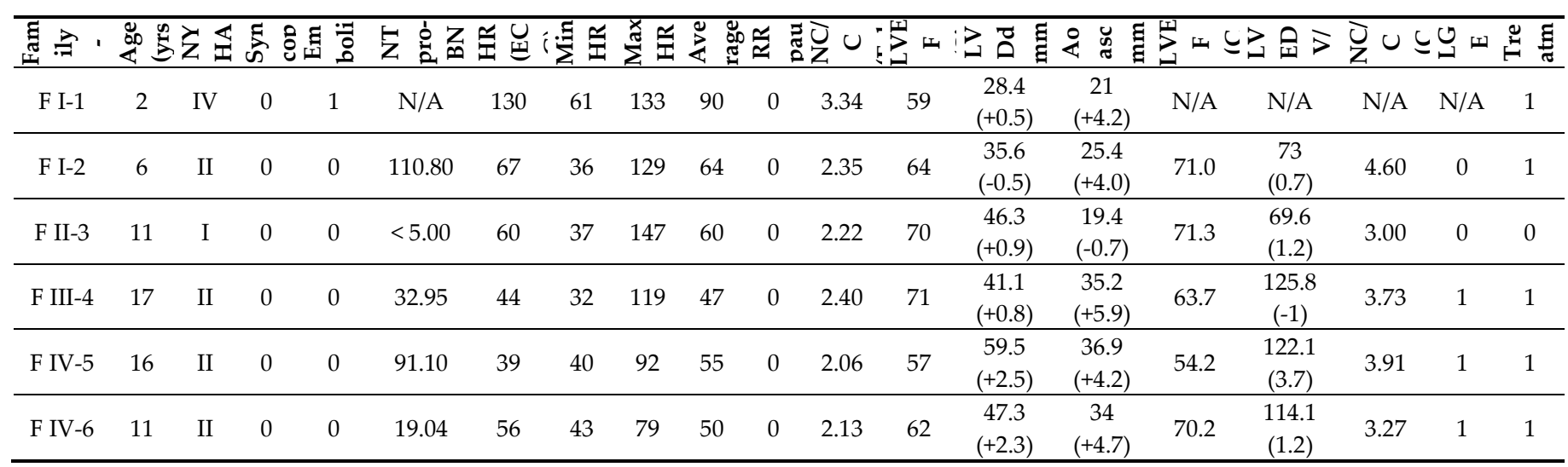

F I-IV: families, 1-6: family members, NYHA- New York Heart Association, yrs-years, NTproBNP - N-terminal probrain natriuretic peptide, HR - heart rate, bpm-beat per minute, ECG - electrocardiogram, Min - minimal, Max - maximal, RR pauses - sinus pauses, sec-seconds, NC/C - noncompacted to compacted myocardial layer ratio, LVEF - left ventricular ejection fraction, LVDd - left ventricular diastolic diameter, Ao asc - ascending aorta, CMR- cardiovascular magnetic resonance, BSA-body surface area, LV EDV - left ventricular end-diastolic volume, LGE - late gadolinium enhancement, N/A - data is not available , " 0 " - were not present, " 1 "- were present.

In echocardiography, the median NC/C ratio in all patients was 2.29 (2.13 - 2.40), which met Jenni's criteria for the diagnosis of LVNC. Dilation of the ascending aorta exceeding the standard indexed to BSA was found in 5 study participants. In 5 of the 6 children CMR imaging was performed in order to further assess cardiac morphology and function, and to screen for features of myocardial fibrosis. In one child, CMR was not performed due to his severe clinical condition, hemodynamic instability and low body weight. Among the 5 patients who underwent CMR, the diagnosis of LVNC was confirmed in all according to Petersen's criteria with the median of NC/C ratio 3.73 (3.14 4.25). All patients studied were diagnosed with sinus bradycardia in accordance with the recommendations in the literature $[12,13]$.

Characteristics of families. The pedigrees of individual families are presented in Figure 1.

\subsection{Family 1.}

Two sisters, 2-years old and 6-years old, diagnosed with LVNC were admitted to our institute for cardiologic evaluation. Family history revealed that their grandfather (father's father) was diagnosed with sinus bradycardia and pacemaker was implanted. However, his echocardiographic examination is not known, and we cannot state whether there were LVNC features. The younger girl (F I-1) diagnosed with LVNC at 3 months of age and progressive heart failure was admitted to the intensive care unit with symptoms of severe heart failure (NYHA group IV) and multi-organ injury On echocardiography, the NC/C ratio was 3.34, LV size was normal but LV systolic function was reduced. In addition, there was a dilation of the ascending aorta. In the resting ECG recording, the heart rate was 130 beats per minute (bpm), while the average daily rhythm in the 24-hour Holter study was $90 \mathrm{bpm}$ (the child was treated with an infusion of catecholamines). In the course of the disease, the child developed ischemic cerebral stroke. The girl died in the mechanism of sinus bradycardia. The second girl (F I-2) underwent cardiological examinations after the diagnosis of LVNC in her sister. Clinical symptoms included dyspnea and fatigue on effort (NYHA class II). The resting ECG showed sinus bradycardia (67 bpm), and in the 24-hour Holter monitoring, the average daily rhythm was $64 \mathrm{bpm}$. Echocardiography revealed $\mathrm{LVNC}$ features with $\mathrm{NC} / \mathrm{C}$ ratio of 2.35 and dilation of the ascending aorta. In CMR the NC/C ratio was 4.60, no LGE was found. In both imaging studies, the LV size and function were within the normal range. ACE inhibitors and spironolactone were included in the treatment. 
Family 1

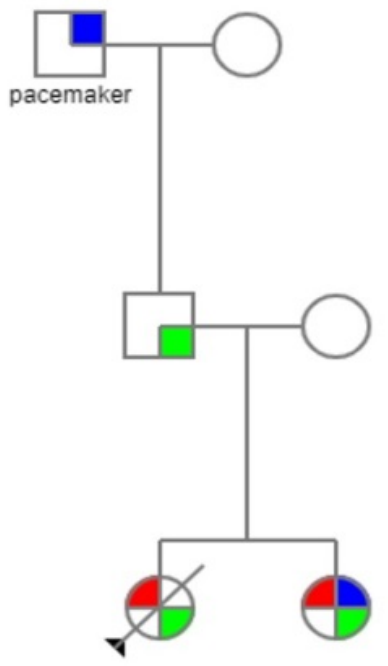

Family 3

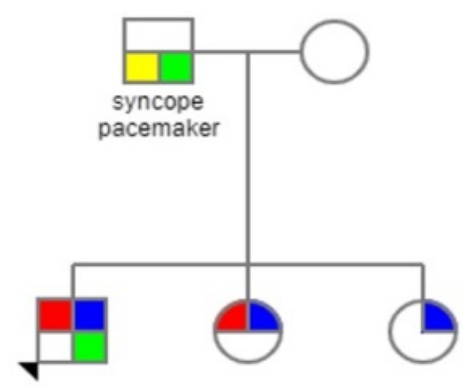

Family 2

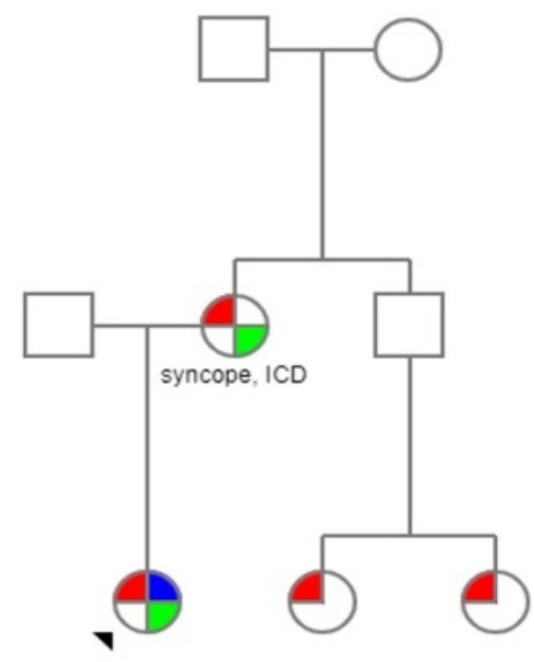

Family 4

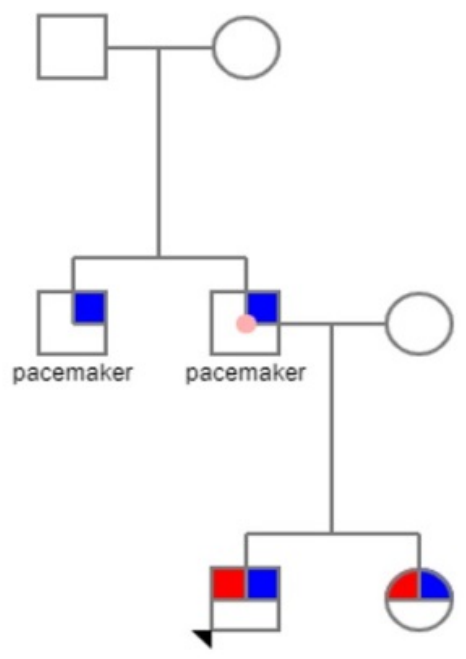

Figure 1. Family pedigrees
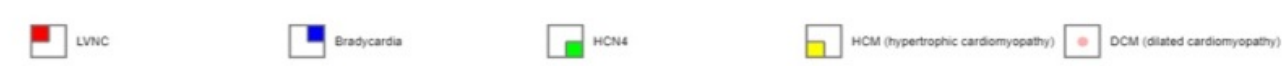

Figure 1. Pedigree of four families with LVNC and sinus bradycardia. Circle - females; square males; arrow - proband. In red - patients affected by left ventricular non-compaction, in blue - bradycardia, in green - HCN4 mutation, in yellow - hypertrophic cardiomyopathy, in pink circle - dilated cardiomyopathy. A strikethrough field - death.

NGS analysis performed in this family in the younger girl revealed a heterozygous known HCN4 molecular variant c.1444G>A p.Gly482Arg that was subsequently confirmed in her affected sister and her father. Nine of the used in silico algorithms (CADD, MetaSVM, MutationAssessor, Polyphen2_HDIV, Polyphen2_HVAR, MetaLR, SIFT, FATHMM, MutationTaster) predicted this change as deleterious. The c.1444G $>$ A variant was not found in the utilized frequency population databases (gnomAD, EVS, UK10K) or in our DMG cohort. It was reported as a disease-causing variant in sinus bradycardia and myocardial noncompaction cases in HGMD (ID: CM159293) and also as pathogenic/likely pathogenic in Brugada syndrome patients in ClinVar (ID: 197253). 


\subsection{Family 2.}

An 11-year-old girl (F II-3) was admitted for cardiac diagnostics due to observed sinus bradycardia and a positive family history of LVNC. Her mother had been diagnosed with LVNC and syncope, and a cardioverter-defibrillator (ICD) was implanted. In addition, her brother's daughters were also diagnosed with LVNC. During hospitalization, the patient did not report any complaints and did not present any symptoms of heart failure (NYHA class I) and bradycardia. The ECG showed sinus bradycardia of $60 \mathrm{bpm}$, while the average 24-hour heart rhythm in the Holter examination was $60 \mathrm{bpm}$. Echocardiography showed the NC/C ratio of 2.22, and the size of the ascending aorta was within the normal range. CMR examination confirmed the diagnosis of LVNC, the NC/C ratio was 3.00, but no LGE was found. In both of the above studies, LV size and systolic function were normal. Pharmacological treatment was not started.

The heterozygous known $\mathrm{HCN} 4$ molecular variant c.1454C $>\mathrm{T}$ p.Ala485Val was identified in the proband and further confirmed in her mother. Eight in silico algorithms (CADD, MetaSVM, Polyphen2_HDIV, Polyphen2_HVAR, MetaLR, SIFT, FATHMM, MutationTaster) predicted this change as deleterious. The c.1454C $>\mathrm{T}$ variant was not found in the EVS, UK10K, and DMG databases, but it was noted in gnomAD with MAF < 0.00001. This variant has no ClinVar submission but appeared in HGMD as a diseasecausing variant in Sinus bradycardia patients (ID: CM104901).

\subsection{Family 3.}

A 17-year-old patient (F III-4) was admitted to our institute due to sinus bradycardia and a family history of bradycardia and cardiomyopathy in other family members. Family history revealed that his sister was diagnosed with LVNC and sinus bradycardia, while the other sister was also treated for bradycardia. The boy's father was diagnosed with hypertrophic cardiomyopathy and a syncope, he had a pacemaker implanted. Patient's clinical symptoms included only dyspnea and fatigue on effort (NYHA class II). Electrocardiogram showed sinus rhythm of $44 \mathrm{bpm}$ (Figure 2).
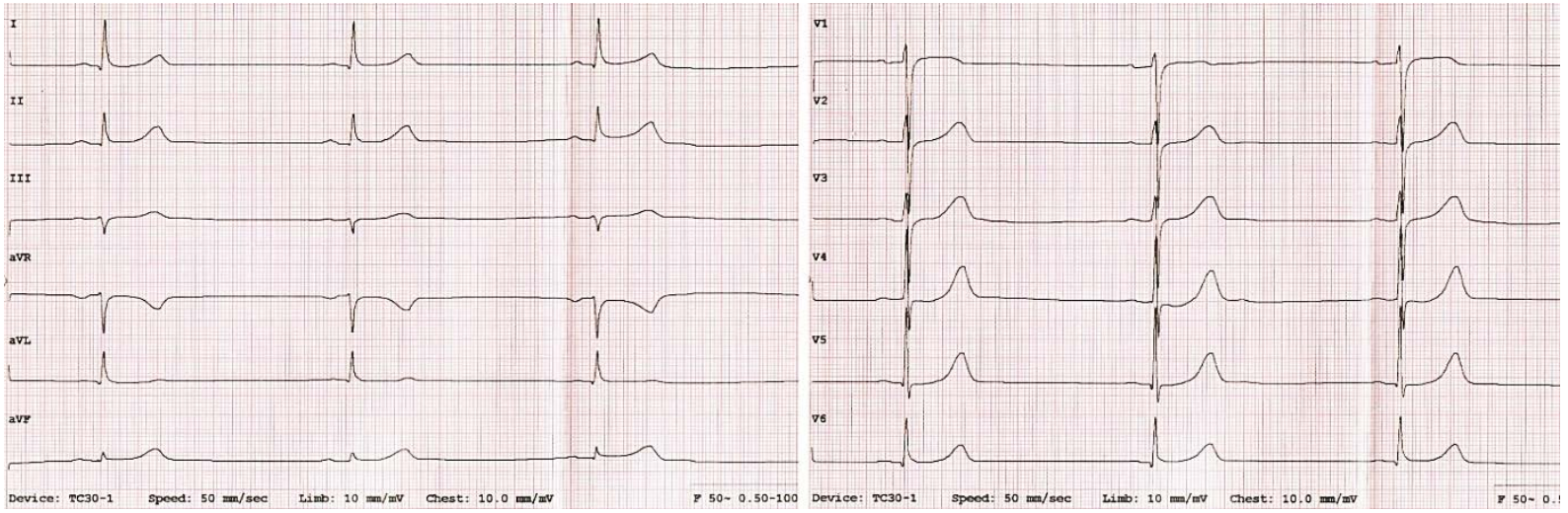

Figure 2. ECG recording shows sinus bradycardia $44 \mathrm{bpm}$ in a 17-year-old patient.

The mean 24-hour heart rate during Holter test was $47 \mathrm{bpm}$. Echocardiography revealed LVNC phenotypic features with the NC/C ratio of 2.40 and dilation of the ascending aorta. In the CMR study, the NC/C ratio was 3.73 and the presence of LGE in the LV myocardial wall was found. Both echocardiography and CMR showed normal LV size and LV systolic function. The patient was receiving treatment for heart failure (ACE inhibitor, spironolactone) and salbutamol for sinus bradycardia.

The heterozygous known HCN4 molecular variant c.1438G>C p.Gly480Arg was found in the proband, and was also identified in his father (DNA from his sisters was not available for testing). Nine in silico algorithms (CADD, MetaSVM, MutationAssessor, Polyphen2_HDIV, Polyphen2_HVAR, MetaLR, SIFT, FATHMM, MutationTaster) predicted this change as deleterious. The c. $1438 \mathrm{G}>\mathrm{C}$ variant was not present in the frequency 
population databases. It was reported as disease-associated polymorphism in sinus bradycardia asymptomatic association in HGMD (ID: CM073122) and was classified as pathogenic in Sick sinus syndrome 2 patient in ClinVar (ID: 5176).

\subsection{Family 4.}

The siblings, a 16-year-old girl (F IV-5) and her 11-year-old brother (F IV-6), were admitted to our Department of Cardiology due to sinus bradycardia. Family history revealed that their father had been diagnosed with sinus bradycardia and dilated cardiomyopathy (DCM); he had a pacemaker implanted. Moreover, their father's brother also had a pacemaker implanted due to sinus bradycardia. The girl was asymptomatic, no syncope was present. She only reported increased fatigue during exercise (NYHA class II). A nodal rhythm of approximately $39 \mathrm{bpm}$ was recorded in the resting ECG. In the 24-hour Holter ECG, the average daily rhythm was $55 \mathrm{bpm}$. Sick sinus syndrome (SSS) and supraventricular premature beats were diagnosed. Echocardiography revealed LVNC with $\mathrm{NC} / \mathrm{C}$ ratio of 2.06 and dilation of the ascending aorta. In the CMR examination, the NC/C ratio was 3.91, foci of LGE were found both in the myocardial wall of the left ventricle and in the wall of the left atrium (Figure 3).

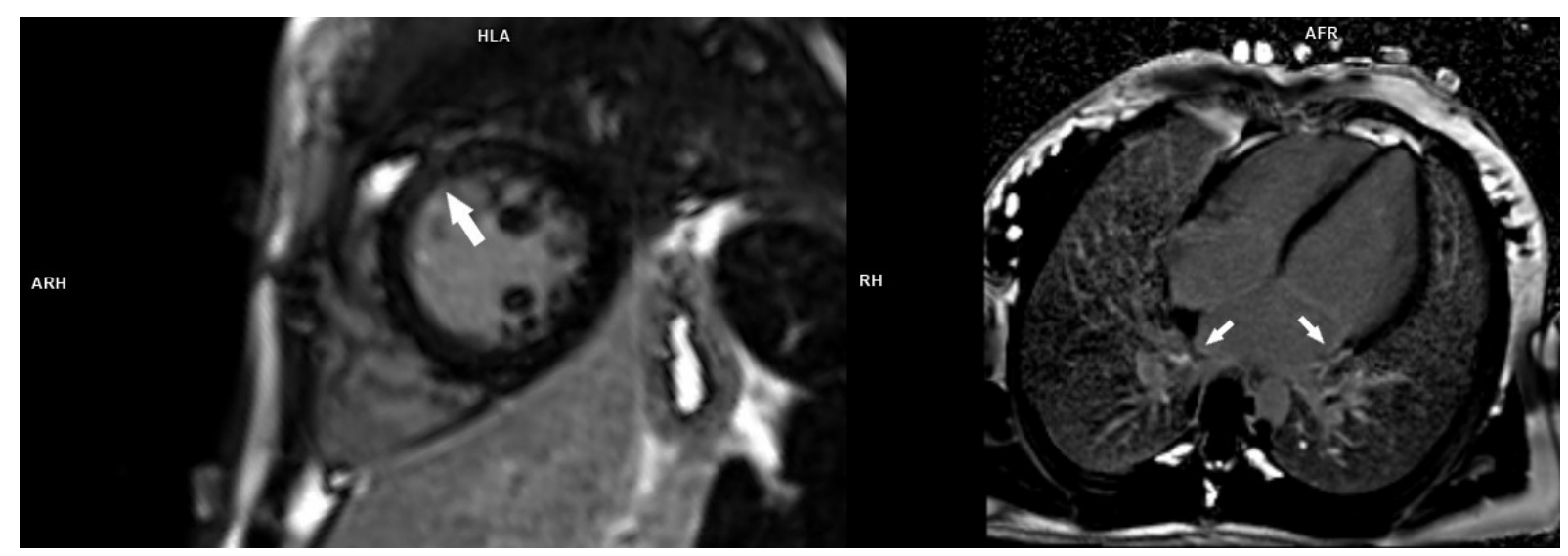

Figure 3. Late gadolinium enhancement (LGE) PSIR images showing midwall LGE in the mid anterior and anteroseptal segments of the left ventricle (A - short-axis view) and in the left atrium (B - 4-chamber view) in a 16-year-old female patient.

In both echocardiography and CMR, LV was enlarged with a borderline systolic function. The girl received treatment for heart failure (ACE inhibitor, spironolactone) and salbutamol due to sinus bradycardia.

The second sibling, the boy, had no symptoms of sinus bradycardia or syncope. Clinical symptoms included dyspnea and fatigue on effort (NYHA class II). The heart rate on the ECG was $56 \mathrm{bpm}$, with the mean daily heart rhythm on the 24-hour Holter study of $50 \mathrm{bpm}$. The patient was diagnosed with sick sinus syndrome and premature supraventricular beats, without episodes of supraventricular tachycardia. Echocardiography revealed typical LVNC features with NC/C ratio of 2.13 and LV enlargement with normal systolic function. There was also a dilation of the ascending aorta. CMR confirmed the diagnosis of LVNC (NC/C ratio was 3.27), while the LV size and systolic function were normal. Like his sister, the boy was found to have LGE in the LV myocardial wall and in the left atrium. The patient is treated with medications such as ACE inhibitors and salbutamol.

Molecular analysis performed in the girl revealed no candidate pathogenic/likely pathogenic variant in the studied cardiomyopathy panel. 


\section{Discussion}

Left ventricular noncompaction cardiomyopathy is a genetically and phenotypically heterogeneous myocardial disease characterized by multiple prominent trabeculations and deep intertrabecular recesses. It is a relatively new, yet rare clinical entity, but nevertheless the third most common cardiomyopathy in childhood and is associated with congestive heart failure, arrhythmias, atrioventricular conduction disorders, sudden cardiac death and/or thromboembolic events. However, patients with isolated LVNC can also be asymptomatic and diagnosed after an abnormal ECG, echocardiogram or family screening [21,22].

In the literature reports, the relationship between LVNC and sinus bradycardia has been described in approximately $55 \%$ of cases, although the basis of conduction disturbances remains unclear $[7,23$,$] . The results of other studies demonstrated that the cause of$ sinus bradycardia in patients with LVNC may be the presence of a pathogenic variant in the HCN4 gene [10,24,25].

Mutations in $\mathrm{HCN} 4$ are thought to predominantly underlie sinoatrial node (SAN) disorders because $\mathrm{HCN} 4$ expression is mostly limited to the cardiac conduction system, especially the SAN area [24]. HCN4 is the most prominent channel in the sinoatrial node being a major determinant of the cardiac pacemaker current (If) and playing a crucial role in the automaticity of the sinus node through the generation of a slow diastolic depolarization during phase 4 of the cardiac action potential. Thus, this is a crucial channel for appropriate pacemaker activity and conduction system function [24,26]. Cardiac pacemaker current channel malfunction due to $\mathrm{HCN} 4$ mutations may result in cardiac function disorders. They have been reported mainly in patients with sick sinus syndrome (SSS) and Brugada syndrome. However, in recent years, a broad spectrum of conditions, including sinus bradycardia, sinus tachycardia, atrial fibrillation, atrioventricular block, idiopathic ventricular tachycardia, left ventricular noncompaction, myocardial infraction, sudden infant death syndrome, arrhythmogenic right ventricular cardiomyopathy, dilation of the aorta and chronotropic incompetence have been noted [24,27].

The epidemiology of HCN4 pathogenic variants in LVNC patients is not fully determined, with limited data concerning especially the pediatric population. This can be due to rare occurrence of childhood LVNC, lack of widespread routine genetic testing, and lack of $H C N 4$ in the applied gene panels. Van Waning et al. [28] collected data from papers published between January 1999 and March 2018 with clinical and molecular characteristics of $561 \mathrm{LVNC}$ cases (adults and children), and found twenty-two subjects (4\%) who carried HCN4 alterations causative for the disease. HCN4 variants accounted for $10 \%$ of (likely) pathogenic variants identified in a cohort of 95 adults diagnosed with LVNC by Richard et al. [29] as well as in a similar group reported on by Cambon-Viala et al. [30]. In a recent study, Hirono et al. [31] found two children with HCN4-related LVNC among 206 patients aged less than 16 years.

Milano et al. were the first to describe the coexistence of LVNC, sinus bradycardia and mutations in the HCN4 gene and emphasized the role of their familial occurrence [10].

Although there are reports showing significant phenotypic heterogeneity in pathogenic $H C N 4$ gene variants, only patients who have the LVNC phenotype have been described. Moreover, the detection of pathogenic variants in patients with LVNC identifies cases (and their family members) who are at risk of developing adverse cardiac events, including arrhythmias [32]..

To date, at least 50 (likely) pathogenic HCN4 molecular variants have been collected in the Human Gene Mutation Database Professional 2021v.3. All molecular variants identified in this study are known changes that affect highly evolutionary conserved amino acids located in close proximity with the essential pore-forming domain of the protein (Figure 4). 
A

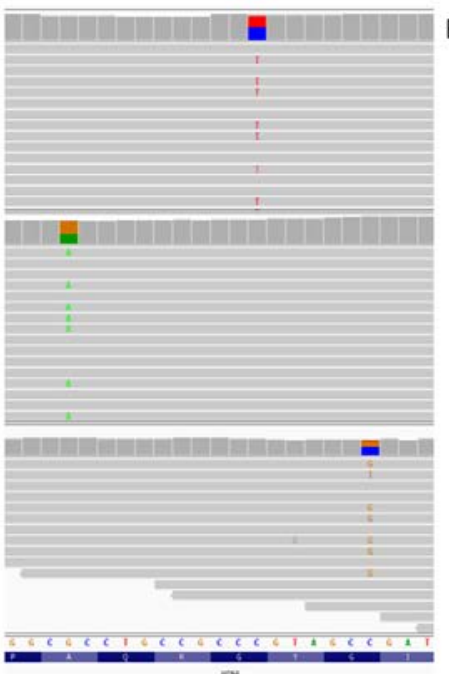

B
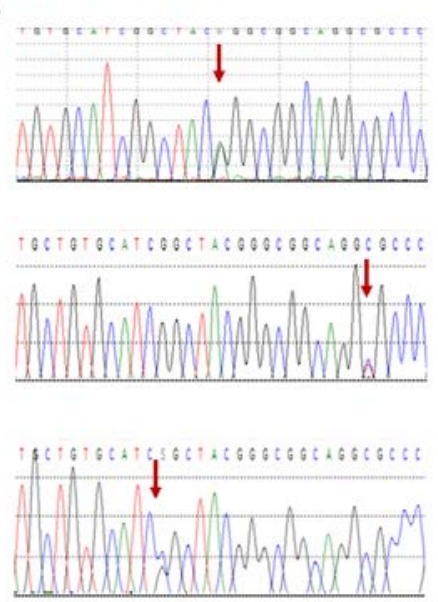

HMLCIGYGRQAPVGMS Homosapiens HMLCIGYGROAPVGMS Mus musculus HMLCGYGRQAPVGMS Rattus norvegicus HMLCGYGROAPVGMS Mesocricetus auratus HMLCIGYGRQAPVGMS Canis lupus familiaris HMLCIGYGROAPMGMS Oryctolagus cuniculus HMLCIGYGQQAPVGMS Gallus gallus HMLCIGYGQQAPMGMS Columba livid HMLCIGYGQQAPEGMT Xenopus tropicalis

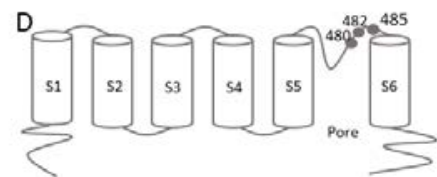

Figure 4. Heterozygous molecular variants c.1444G>A, c.1454C>T, and c.1438G>C identified in this study: (A) NGS results displayed in Integrative Genomics Viewer (http://software.broadinstitute.org/software/igv/); (B) Sanger sequencing electropherograms. Arrows indicate an altered nucleotide; C/ Multiple alignment of HCN4 region of interest, showing amino acid residues conserved between species (bold font); (D) Schematic topology of HCN4, with the altered amino acids indicated. The S1-S6 blocks denote structural segments with the pore-forming region comprising amino acids in positions $465-$ 486.

These variants have been reported previously, mainly in adults with a complex phenotype of LVNC and sinus bradycardia and myocardial noncompaction [25,29,30,33,34], while pediatric presentations are rare. Familial forms of SSS caused by HCN4 mutations rarely manifest themselves clinically in childhood and are mainly recognized after adolescence. Only a few literature descriptions of teenage patients manifested with a complex phenotype of sinus bradycardia and LVNC can be found in literature. Ishikawa et al. described a case of a 13-year-old girl with significant sinus bradycardia requiring permanent pacing at the age of 17 years [34]. Millat et al. [25] reported on a family with three sisters $(12,18$ and 24 years old) who presented with a similar phenotype: sinus bradycardia in combination with LVNC. In our study, we present early onset of clinical symptoms of the disease. Both siblings from Family 1 were diagnosed before 6 years of age, with severe multi-organ dysfunction and ischemic cerebral stroke resulting in death at 2 years in one of them.

Different base substitution at the same HCN4 nucleotide position (c.1438, c.1444, and c.1454) leading to the same or various amino acid changes has been reported in multiple studies $[10,26,29,30]$, highlighting the important role of this region for proper function of the HCN4 channel. Functional effect of these variants has been studied extensively, demonstrating that the p.Gly480Arg, p.Gly482Arg and p.Ala485Val variants severely abolish pacemaker currents with a hyperpolarization shift in voltage dependence of activation $[10,26,35,36]$. Recent literature reports indicate that the molecular etiology of LVNC is detected in approximately $40 \%$ of cases [37]. As in the reports of other authors [10,25], in our patients a significant relationship between LVNC, sinus bradycardia and HCN4 mutation has been demonstrated. The molecular etiology of heart abnormalities was confirmed in three out of four families (a total of 4 patients). It should be emphasized that in Family 4, despite the typical course of LVNC and sinus bradycardia, no molecular cause was identified.

A very important aspect of the clinical evaluation of our patients is to demonstrate the presence of dilation of the ascending aorta. There are only a few studies showing an association of mutations in the HCN4 gene with dilation of the ascending aorta. As reported by Vermeer et al., evidence was provided that dilation of the ascending aorta also constitutes a part of the clinical spectrum of HCN4 mutations. Moreover, these authors 
demonstrated that $\mathrm{HCN} 4$ mutation carriers have aortic dilation with age, which undoubtedly influences the prognosis and cardiac care in this group of patients [38].

According to the literature the presence of LGE in the LV myocardium is found in $40 \%$ of LVNC patients [39]. In our study, out of 5 patients who underwent CMR examination, $3(60 \%)$ children showed LGE in the LV myocardium and in the left atrium. The presence of LV fibrosis is a strong independent predictor of poor prognosis, not only in LVNC but also in another form of childhood cardiomyopathy, i.e. hypertrophic cardiomyopathy [40]. The results of published studies show that the prognosis worsens in patients with LVNC and the presence of LGE and/or global impairment of LV systolic function [41,42]. These authors also emphasize that only hypertrabeculation meeting current LVNC diagnostic criteria has no significant impact on patient prognosis, but when the LVNC pattern is associated with a DCM-like phenotype and/or LGE, the prognosis deteriorates significantly. Among our 3 patients with LVNC and the presence of LGE, two children had LV enlargement requiring further regular cardiological control.

The literature presents the results of a study showing that atrial fibrosis, quantified with CMR-LGE, is an important predictor of clinically significant sinus node dysfunction and sinus bradycardia requiring pacemaker implantation [43]. It should be especially emphasized that in our group of 3 children with LVNC and LGE, two had LGE both in the LV wall and within the left atrial wall. These patients were diagnosed with sinus node dysfunction and sinus bradycardia, and further investigation was scheduled prior to qualification for permanent pacing implantation.

In summary, systematic search for HCN4 mutations should be undertaken in patients with such a complex clinical picture. Additionally, we emphasized the role of CMR in LVNC, not only regarding diagnosis but also risk stratification and future medical treatment. This is important for everyday clinical practice and for further patient care as it enables targeted treatment and further management.

\section{Conclusions}

1. The HCN4 mutation influences the presence of a complex LVNC phenotype, sinus bradycardia and dilation of the ascending aorta.

2. HCN4 mutation may be associated with early presentation of clinical symptoms and severe course of the disease.

3. It is particularly important to assess myocardial fibrosis not only within the ventricles, but also in the atria in patients with LVNC and sinus bradycardia.

Supplementary Materials: Supplementary Material: Cardiomyopathy-related genes analyzed in this study.

Author Contributions: Conceptualization: LZ, AP, DPA; Methodology: DPA, AP, LZ, AMR, JS, GK, EC, MB; Validation: LZ, DPA, AMR, JS, MB; Formal Analysis: AP, DPA, LZ, DW; Investigation: AP, DPA, LZ, AMR, JS, GK, EC, DW; Resources: AP, LZ, DPA, JS, MB; Data Curation, AP, DPA, LZ, AMR, JS, EC, MB; Writing - Original Draft Preparation- AP, DPA, LZ, MB, JS; Writing - Review \& Editing, LZ, AP, DPA; Supervision- LZ. All authors have read and agreed to the published version of the manuscript.

Funding: This work was partially founded by The Children's Memorial Health Institute (statutory grant no. S177/2018)

Institutional Review Board Statement: The study was conducted according to the guidelines of the Declaration of Helsinki and approved by the Institutional Ethics Committee of The Children's Memorial Health Institute (protocol code 45/KBE/2018 and date of approval 24/Oct/2018).

Informed Consent Statement: Informed consent was obtained from all subjects and their parents involved in the study.

Data Availability Statement: The data presented in this study are available on request from the corresponding author. 


\begin{abstract}
Acknowledgments: In this section, you can acknowledge any support given which is not covered by the author contribution or funding sections. This may include administrative and technical support, or donations in kind (e.g., materials used for experiments).
\end{abstract}

Conflicts of Interest: The authors declare no conflict of interest. The funders had no role in the design of the study; in the collection, analyses, or interpretation of data; in the writing of the manuscript, or in the decision to publish the results.

\title{
References
}

1. Tsai, SF.; Ebenroth, ES.; Hurwitz, RA.; Cordes, TM.; Schamberger, MS.; Batra, AS. Is left ventricular noncompaction in children truly an isolated lesion? Pediatr Cardiol, 2009, 30: 597-602.

2. Ergul, Y.; Nisli, K.; Demirel, A.; Varkal, MA.; Oner, N.; Dursun, M.; Dindar, A.; Aydogan, U.; Omeroglu, RE. Left ventricular non-compaction in children and adolescents: clinical features, treatment and follow-up. Cardiol J. 2011,18(2):176-84.

3. Miszalski-Jamka, K.; Jefferies, J.L.; Mazur, W.; Głowacki, J.; Hu, J.; Lazar, M.; Gibbs, R.A.; Liczko, J.; Kłyś, J.; Venner, E.; et al. Novel Genetic Triggers and Genotype-Phenotype Correlations in Patients With Left Ventricular Noncompaction. Circ Cardiovasc Genet. 2017, 10(4):e001763.

4. Oechslin, E.; Jenni R. Left ventricular non-compaction revisited: a distinct phenotype with genetic heterogeneity? Eur Heart J. 2011, 32:1446-1456.

5. Hoedemaekers, Y.M.; Caliskan, K.; Majoor-Krakauer, D.F. Clinical Features of Noncompaction Cardiomyopathy. Departments of Clinical Genetics and Cardiology, Erasmus, MC, Rotterdam 2012.

6. Gupta, U.; Makhija, P. Left Ventricular Noncompaction Cardiomyopathy in Pediatric Patients: A Case Series of a Clinically Heterogeneous Disease. Pediatr Cardiol. 2017, 38(4):681-690.

7. Celiker, A.; Ozkutlu, S.; Dilber, E.; Karagöz, T. Rhythm abnormalities in children with isolated ventricular noncompaction. Pacing Clin Electrophysiol. 2005, 28(11):1198-202.

8. Egan, K.R.; Ralphe, J.C.; Weinhaus, L.; Maginot, K.R. Just sinus bradycardia or something more serious? Case Rep Pediatr. 2013, $1-5$

9. S. Ozkutlu, S.; Onderoglu, L.; Karagöz, T.; Celiker, A.; Sahiner U.M. Isolated noncompaction of left ventricular myocardium with fetal sustained bradycardia due to sick sinus syndrome. Turk J Pediatr. 2006, 48: 383-386.

10. Milano, A.; Vermeer, A.M.; Lodder, E.M.; Barc, J.; Verkerk, A.O.; Postma, A.V.; van der Bilt, I.A.; Baars, M.J.; van Haelst, P.L.; Caliskan, K.; et al. HCN4 mutations in multiple families with bradycardia and left ventricular noncompaction cardiomyopathy. J Am Coll Cardiol. 2014, 64(8):745-56.

11. Jenni, R.; Oechslin, E.; Schneider, J.; Attenhofer Jost, C.; Kaufmann, PA. Echocardiographic and pathoanatomical characteristics of isolated left ventricular non-compaction: a step towards classification as a distinct cardiomyopathy. Heart. 2001, 86(6): 66671.

12. Fleming, S.; Thompson, M.; Stevens, R.; Heneghan, C.; Plüddemann, A.; Maconochie, I.; Tarassenko, L.; Mant, D. Normal ranges of heart rate and respiratory rate in children from birth to 18 years of age: a systematic review of observational studies. Lancet. 2011, 19; 377(9770):1011-8.

13. Baruteau, AE.; Perry, JC.; Sanatani, S.; Horie, M.; Dubin, AM. Evaluation and management of bradycardia in neonates and children. Eur J Pediatr. 2016,175(2):151-61.

14. Kampmann, C.; Wiethoff, C.M.; Wenzel, A.; Stolz, G.; Betancor, M.; Wippermann, C.F.; Huth, R.G.; Habermehl, P.; Knuf, M.; Emschermann, T.; Stopfkuchen, H. Normal values of M mode echocardiographic measurements of more than 2000 healthy infants and children in central Europe. Heart. 2000, 83(6):667-72.

15. Chubb, H.; Simpson, J.M. The use Z-score in paediatric cardiology. Ann Pediatr Cardiol. 2012, 5(2):179-184.

16. Gautier, M.; Detaint, D.; Fermanian, C.; Aegerter, P.; Delorme, G.; Arnoult, F.; Milleron, O.; Raoux, F.; Stheneur, C.; Boileau, C.; Vahanian, A.; Jondeau, G. Nomograms for aortic root diameters in children using two-dimensional echocardiography. Am J Cardiol. 2010, 15;105(6):888-94.

17. Petersen, S.E.; Selvanayagam, J.B.; Wiesmann, F.; Robson, M.D.; Francis, J.M.; Anderson, R.H.; Watkins, H.; Neubauer, S. Left ventricular non-compaction: Insights from cardiovascular magnetic resonance imaging. J. Am. Coll. Cardiol. 2005, 46, 101-105.

18. Reiter, G.; Reiter, U.; Rienmuller, R.; Gagarina, N.; Ryabikin, A. On the value of geometry-based models for left ventricular volumetry in magnetic resonance imaging and electron beam tomography: a Bland-Altman analysis. Eur J Radiol. 2004, 52:1108 .

19. van der Ven, J.P.G.; Sadighy, Z.; Valsangiacomo Buechel, E.R.; Sarikouch, S.; Robbers-Visser, D.; Kellenberger, C.J.; Kaiser, T.; Beerbaum, P.; Boersma, E.; Helbing, W.A. Multicentre reference values for cardiac magnetic resonance imaging derived ventricular size and function for children aged 0-18 years. Eur Heart J Cardiovasc Imaging. 2019, 89:109.

20. Lipiński, P.; Ciara, E.; Jurkiewicz, D.; Płoski, R.; Wawrzynowicz-Syczewska, M.; Pawłowska, J.; Jankowska, I. Progressive familial intrahepatic cholestasis type 3: Report of four clinical cases, novel ABCB4 variants and long-term follow-up. Ann Hepatol. 2021, 25:100342.

21. Brescia, S.T.; Rossano, J.W.; Pignatelli, R.; Jefferies, J.L.; Price, J.F.; Decker, J.A.; Denfield, S.W.; Dreyer, W.J.; Smith, O.; Towbin, J.A.; Kim, J.J. Mortality and sudden death in pediatric left ventricular noncompaction in a tertiary referral center. Circulation. 2013, 4;127(22):2202-8. 
22. Kobza, R.; Jenni, R.; Erne, P.; Oechslin, E.; Duru, F. Implantable cardioverter-defibrillators in patients with left ventricular noncompaction. Pacing Clin Electrophysiol. 2008, 31(4):461-467.

23. Salerno, J.C.; Chun, T.U.; Rutledge, J.C. Sinus bradycardia, Wolff Parkinson White, and left ventricular noncompaction: an embryologic connection? Pediatr Cardiol. 2008, 29(3):679-682.

24. Servatius, H.; Porro, A.; Pless, S.A.; Schaller, A.; Asatryan, B.; Tanner, H.; de Marchi, S.F.; Roten, L.; Seiler, J.; Haeberlin, A.; et al. Phenotypic Spectrum of HCN4 Mutations: A Clinical Case. Circ Genom Precis Med. 2018, 11(2):e002033.

25. Millat, G.; Janin, A.; de Tauriac, O.; Roux, A.; Dauphin, C. HCN4 mutation as a molecular explanation on patients with bradycardia and non-compaction cardiomyopathy. Eur J Med Genet. 2015, 58(9):439-42.

26. Schweizer, P.A.; Schröter, J.; Greiner, S.; Haas, J.; Yampolsky, P.; Mereles, D.; Buss, S.J.; Seyler, C.; Bruehl, C.; Draguhn, A.; Koenen, M.; Meder, B.; Katus, H.A.; Thomas, D. The symptom complex of familial sinus node dysfunction and myocardial noncompaction is associated with mutations in the HCN4 channel. J Am Coll Cardiol. 2014, 26;64(8):757-67.

27. Towbin, J.A. Ion channel dysfunction associated with arrhythmia, ventricular noncompaction, and mitral valve prolapse: a new overlapping phenotype. J Am Coll Cardiol. 2014, 26;64(8):768-71.

28. van Waning, J.I.; Moesker, J.; Heijsman, D.; Boersma, E.; Majoor-Krakauer, D. Systematic Review of Genotype-Phenotype Correlations in Noncompaction Cardiomyopathy. J Am Heart Assoc. 2019, 8(23):e012993.

29. Richard, P.; Ader, F.; Roux, M.; Donal, E.; Eicher, J.C.; Aoutil, N.; Huttin, O.; Selton-Suty, C.; Coisne, D.; Jondeau, G.; et al. Targeted panel sequencing in adult patients with left ventricular non-compaction reveals a large genetic heterogeneity. Clin Genet. 2019, 95(3):356-367.

30. Cambon-Viala, M.; Gerard, H.; Nguyen, K.; Richard, P.; Ader, F.; Pruny, J.F.; Donal, E.; Eicher, J.C.; Huttin, O.; Selton-Suty, C.; et al. Phenotype/Genotype Relationship in Left Ventricular Noncompaction: Ion Channel Gene Mutations Are Associated With Preserved Left Ventricular Systolic Function and Biventricular Noncompaction: Phenotype/Genotype of Noncompaction. J Card Fail. 2021, 27(6):677-681.

31. Hirono, K.; Hata, Y.; Miyao, N.; Okabe, M.; Takarada, S.; Nakaoka, H.; Ibuki, K.; Ozawa, S.; Origasa, H.; Nishida, N.; Ichida, F. LVNC study collaborates. Increased Burden of Ion Channel Gene Variants Is Related to Distinct Phenotypes in Pediatric Patients With Left Ventricular Noncompaction. Circ Genom Precis Med. 2020, 13(4):e002940.

32. Mazzarotto, F.; Hawley, M.H.; Beltrami, M.; Beekman, L.; de Marvao, A.; McGurk, K.A.; Statton, B.; Boschi, B.; Girolami, F.; Roberts, A.M.; et al. Systematic large-scale assessment of the genetic architecture of left ventricular noncompaction reveals diverse etiologies. Genet Med. 2021, 23(5):856-864.

33. Chanavat, V.; Janin, A.; Millat, G. A fast and cost-effective molecular diagnostic tool for genetic diseases involved in sudden cardiac death. Clin Chim Acta. 2016, 30;453:80-5.

34. Ishikawa, T.; Ohno, S.; Murakami, T.; Yoshida, K.; Mishima, H.; Fukuoka, T.; Kimoto, H.; Sakamoto, R.; Ohkusa, T.; Aiba, T.; et al. Sick sinus syndrome with HCN4 mutations shows early onset and frequent association with atrial fibrillation and left ventricular noncompaction. Heart Rhythm. 2017, 14(5):717-724.

35. Laish-Farkash, A.; Glikson, M.; Brass, D.; Marek-Yagel, D.; Pras, E.; Dascal, N.; Antzelevitch, C.; Nof, E.; Reznik, H.; Eldar, M.; Luria, D. A novel mutation in the HCN4 gene causes symptomatic sinus bradycardia in Moroccan Jews. J Cardiovasc Electrophysiol. 2010, 21(12):1365-72.

36. Nof, E.; Luria, D.; Brass, D.; Marek, D.; Lahat, H.; Reznik-Wolf, H.; Pras, E.; Dascal, N.; Eldar, M.; Glikson, M. Point mutation in the HCN4 cardiac ion channel pore affecting synthesis, trafficking, and functional expression is associated with familial asymptomatic sinus bradycardia. Circulation. 2007, 31;116(5):463-70.

37. Dong, X.; Fan, P.; Tian, T.; Yang, Y.; Xiao, Y.; Yang, K.; Liu, Y.; Zhou, X. Recent advancements in the molecular genetics of left ventricular noncompaction cardiomyopathy. Clin Chim Acta. 2017, 465:40-44.

38. Vermeer, A.M.C.; Lodder, E.M.; Thomas, D.; Duijkers, F.A.M.; Marcelis, C.; van Gorselen, E.O.F.; Fortner, P.; Buss, S.J.; Mereles, D.; Katus, H.A.; et al. Dilation of the Aorta Ascendens Forms Part of the Clinical Spectrum of HCN4 Mutations. J Am Coll Cardiol. 2016, 17;67(19):2313-2315.

39. Wan, J.; Zhao, S.; Cheng, H.; Lu, M.; Jiang, S.; Yin, G.; Gao, X.; Yang, Y. Varied distributions of late gadolinium enhancement found among patients meeting cardiovascular magnetic resonance criteria for isolated left ventricular non-compaction. J Cardiovasc Magn Reson. 2013, 20;15(1):20.

40. Petryka-Mazurkiewicz, J.; Ziolkowska, L.; Kowalczyk-Domagala, M.; Mazurkiewicz, L.; Boruc, A.; Spiewak, M.; Misko, J.; Bieganowska, K.; Marczak, M.; Brzezinska-Rajszys, G. LGE for Risk Stratification in Primary Prevention in Children With HCM. JACC Cardiovasc Imaging. 2020,13(12):2684-2686.

41. Andreini, D.; Pontone, G.; Bogaert, J.; Roghi, A.; Barison, A.; Schwitter, J.; Mushtaq, S.; Vovas, G.; Sormani, P.; Aquaro, G.D.; et al. Long-Term Prognostic Value of Cardiac Magnetic Resonance in Left Ventricle Noncompaction: A Prospective Multicenter Study. J Am Coll Cardiol. 2016, 15;68(20):2166-2181.

42. Grigoratos, C.; Barison, A.; Ivanov, A.; Andreini, D.; Amzulescu, MS.; Mazurkiewicz, L.; De Luca, A.; Grzybowski, J.; Masci, P.G.; Marczak, M.; et al. Meta-Analysis of the Prognostic Role of Late Gadolinium Enhancement and Global Systolic Impairment in Left Ventricular Noncompaction. JACC Cardiovasc Imaging. 2019, 12 (11 Pt 1):2141-2151.

43. Akoum, N.; McGann, C.; Vergara, G.; Badger, T.; Ranjan, R.; Mahnkopf, C.; Kholmovski, E.; Macleod, R.; Marrouche, N. Atrial fibrosis quantified using late gadolinium enhancement MRI is associated with sinus node dysfunction requiring pacemaker implant. J Cardiovasc Electrophysiol. 2012, 23(1):44-50. 